\title{
LINEAR RIGHT IDEAL NEARRINGS
}

\author{
KENNETH D. MAGILL JR.
}

(Received 16 February 2001)

\begin{abstract}
We determine, up to isomorphism, all those topological nearrings $\mathcal{N}_{n}$ whose additive groups are the $n$-dimensional Euclidean groups, $n>1$, and which contain $n$ onedimensional linear subspaces $\left\{J_{i}\right\}_{i=1}^{n}$ which are also right ideals of the nearring satisfying several additional properties. Specifically, for each $w \in \mathcal{N}_{n}$, we require that there exist $w^{i} \in J_{i}, 1 \leq i \leq n$, such that $w=w^{1}+w^{2}+\cdots+w^{n}$ and multiplication on the left of $w$ yields the same result as multiplication by the same element on the left of $w^{n}$. That is, $v w=v w^{n}$ for each $v \in \mathcal{N}_{n}$.
\end{abstract}

2000 Mathematics Subject Classification. 16Y30, 54H13.

1. Introduction. A topological nearring $\mathcal{N}$ here is a triplet $(N,+, \cdot)$ where $(N,+)$ is a (not necessarily commutative) topological group, $(N, \cdot)$ is a topological semigroup and the following right distributive law holds:

$$
(u+v) w=u w+v w \quad \forall u, v, w \in N .
$$

In particular, our nearrings are right nearrings. For information about abstract nearrings, one should consult [2, 4, 5]. An $n$-dimensional Euclidean nearring is any topological nearring whose additive group is the $n$-dimensional Euclidean group $\mathbb{R}^{n}$. The main result of this paper is in Section 2 where we determine, up to isomorphism, for $n>1$, all $n$-dimensional Euclidean nearrings $\mathcal{N}_{n}$ which contain $n$ distinct one-dimensional linear subspaces $\left\{J_{i}\right\}_{i=1}^{n}$ which are also right ideals of $\mathcal{N}_{n}$ such that for each $w \in \mathcal{N}_{n}$, there exist $w^{i} \in J_{i}, 1 \leq i \leq n$, such that $w=w^{1}+w^{2}+\cdots+w^{n}$ and $v w=v w^{n}$ for all $v \in \mathcal{N}_{n}$. As a corollary to the latter result, we obtain the fact that a two-dimensional Euclidean nearring $\mathcal{N}_{2}$ has two distinct nonzero proper closed, connected right ideals $J_{1}$ and $J_{2}$ such that for every $w \in \mathcal{N}_{2}$, there exist $w^{1} \in J_{1}$ and $w^{2} \in J_{2}$ such that $w=w^{1}+w^{2}$ and $v w=v w^{2}$ for all $v \in \mathcal{N}_{2}$ if and only if $\mathcal{N}_{2}$ is isomorphic to one of the seven two-dimensional Euclidean nearrings whose multiplications follow:

(1) $v w=v$ for all $v, w \in \mathcal{N}_{2}$,

(2) $v w=0$ for all $v, w \in \mathcal{N}_{2}$,

(3) $v w=\left(v_{1}, 0\right)$ for all $v, w \in \mathcal{N}_{2}$,

(4) $v w=\left(0, v_{2}\right)$ for all $v, w \in \mathcal{N}_{2}$,

(5) $v w=\left(v_{1}\left|w_{2}\right|^{r}, v_{2} w_{2}\right)$

(6) $v w= \begin{cases}\left(v_{1} w_{2}^{r}, v_{2} w_{2}\right) & \text { for } w_{2} \geq 0, \\ \left(-v_{1}\left|w_{2}\right|^{r}, v_{2} w_{2}\right) & \text { for } w_{2}<0,\end{cases}$ 
(7) $v w= \begin{cases}\left(v_{1}\left(a w_{2}\right)^{r}, a v_{2} w_{2}\right) & \text { for } w_{2} \geq 0, \\ \left(v_{1}\left(b w_{2}\right)^{r}, b v_{2} w_{2}\right) & \text { for } w_{2}>0,\end{cases}$ where $r>0$ in each case, $a \leq 0, b \geq 0$, and $a^{2}+b^{2} \neq 0$.

2. The main theorem. An element $w \in \mathbb{R}^{n}$ will be represented as a row vector, that is, $w=\left(w_{1}, w_{2}, \ldots, w_{n}\right)$ and it will be assumed throughout the remainder of the paper that $n>1$. For $w^{i} \in J_{i}$ where $J_{i}$ is any subset of $\mathcal{N}_{n}$, we have $w^{i}=\left(w_{1}^{i}, w_{2}^{i}, \ldots, w_{n}^{i}\right)$. Furthermore, for all $v, w \in \mathcal{N}_{n}$, the $i$ th coordinate of the product $v w$ will be denoted by $(v w)_{i}$. In general, the function which maps all of a space $X$ into a single point $x$ will be denoted by $\langle x\rangle$. Its domain will be apparent from context. Finally, for $r>0$, we define $0^{r}=0$.

DEFINITION 2.1. A linear right ideal nearring is any $n$-dimensional Euclidean nearring which contains $n$ distinct right ideals $\left\{J_{i}\right\}_{i=1}^{n}$, each of which is a one-dimensional linear subspace of $\mathbb{R}^{n}$, such that for all $w \in \mathcal{N}_{n}$ there exist $w^{i} \in J_{i}, 1 \leq i \leq n$, such that $w=w^{1}+w^{2}+\cdots+w^{n}$ and $v w=v w^{n}$ for all $v \in \mathcal{N}_{n}$.

THEOREM 2.2. An n-dimensional Euclidean nearring $\mathcal{N}_{n}$ is a linear right ideal nearring if and only if $\mathcal{N}_{n}$ is isomorphic to one of the four types of nearrings whose multiplications follow:

$$
\begin{aligned}
& (v w)_{i}=0 \quad \text { or } \quad(v w)_{i}=v_{i} \quad \text { for } 1 \leq i \leq n, \\
& (v w)_{i}=v_{i}\left|w_{n}\right|^{r_{i}} \quad \text { for } i \neq n, \quad(v w)_{n}=v_{n} w_{n},
\end{aligned}
$$

where $r_{i}>0$ for $1 \leq i<n$,

$$
(v w)_{i}= \begin{cases}v_{i}\left(w_{n}\right)^{r_{i}} & \text { for } w_{n} \geq 0, \\ -v_{i}\left|w_{n}\right|^{r_{i}} & \text { for } w_{n}<0,\end{cases}
$$

for $i \neq n$ and $(v w)_{n}=v_{n} w_{n}$ where $r_{i}>0$,

$$
\begin{aligned}
& (v w)_{i}= \begin{cases}v_{i}\left(a w_{n}\right)^{r_{i}} & \text { for } w_{n} \leq 0, i \neq n, \\
v_{i}\left(b w_{n}\right)^{r_{i}} & \text { for } w_{n}>0, i \neq n,\end{cases} \\
& (v w)_{n}= \begin{cases}a v_{n} w_{n} & \text { for } w_{n} \leq 0, \\
b v_{n} w_{n} & \text { for } w_{n}>0,\end{cases}
\end{aligned}
$$

where $r_{i}>0, a \leq 0, b \geq 0$, and $a^{2}+b^{2} \neq 0$.

It is convenient to prove Theorem 2.2 via a sequence of lemmas. Recall that the constant function which maps all of $\mathbb{R}^{n}$ into the real number $r$ is denoted by $\langle r\rangle$ and let $K_{i}=\left\{v \in \mathcal{N}_{n}: v_{j}=0\right.$ for $\left.j \neq i\right\}$.

LEMMA 2.3. The nearring $\mathcal{N}_{n}$ is an $n$-dimensional Euclidean nearring such that each $K_{i}, 1 \leq i \leq n$, is a right ideal of $\mathcal{N}_{n}$ and $v w=v\left(0,0, \ldots, 0, w_{n}\right)$ for all $v, w \in \mathcal{N}_{n}$ if and only if there exist $n$ continuous selfmaps $\left\{f_{i}\right\}_{i=1}^{n}$ of the real numbers $\mathbb{R}$ such that

$$
f_{i}\left(x f_{n}(y)\right)=f_{i}(x) f_{i}(y) \quad \text { for } 1 \leq i \leq n,
$$


and the multiplication on $\mathcal{N}_{n}$ is given by

$$
(v w)_{i}=v_{i} f_{i}\left(w_{n}\right) \quad \text { for } 1 \leq i \leq n .
$$

Proof. Suppose first that there exist $n$ continuous selfmaps of $\mathbb{R}$ satisfying (2.5) and the multiplication in $\mathcal{N}_{n}$ is given by (2.6). We must show that multiplication is associative and that each $K_{i}$ is a right ideal of $\mathcal{N}_{n}$. For $1 \leq i \leq n$, we have

$$
\begin{aligned}
((u v) w)_{i} & =(u v)_{i} f_{i}\left(w_{n}\right)=u_{i} f_{i}\left(v_{n}\right) f_{i}\left(w_{n}\right) \\
& =u_{i} f_{i}\left(v_{n} f_{i}\left(w_{n}\right)\right)=u_{i} f_{i}\left((v w)_{n}\right)=(u(v w))_{i}
\end{aligned}
$$

and we see that multiplication is associative. It follows immediately from (2.6) that each $K_{i}$ is a right ideal of $\mathcal{N}_{n}$.

Suppose, conversely, that $\mathcal{N}_{n}$ is an $n$-dimensional Euclidean nearring such that each $K_{i}, 1 \leq i \leq n$, is a right ideal of $\mathcal{N}_{n}$ and $v w=v\left(0,0, \ldots, 0, w_{n}\right)$ for all $v, w \in \mathcal{N}_{n}$. We must show that there exist $n$ continuous selfmaps $\left\{f_{i}\right\}_{i=1}^{n}$ of the real numbers $\mathbb{R}$ such that (2.5) and (2.6) are satisfied. According to [3, Theorem 2.10] there exist $n^{2}$ continuous functions $\left\{g_{i j}\right\}, 1 \leq i, j \leq n$, from $\mathbb{R}^{n}$ to $\mathbb{R}$ such that

$$
(v w)_{i}=\sum_{j=1}^{n} v_{j} g_{i j}(w) \quad \forall v, w \in \mathbb{R}^{n}
$$

Let $e^{i}$ be a vector such that $e_{j}^{i}=0$ for $j \neq i$ and $e_{i}^{i}=1$. From (2.8), we get

$$
\left(e^{k} w\right)_{i}=\sum_{j=1}^{n} e_{j}^{k} g_{i j}(w)=g_{i k}(w) .
$$

Since $J_{k}$ is a right ideal of $\mathcal{N}_{n}$, we have $e^{k} w \in J_{k}$ for all $w \in \mathcal{N}_{n}$. It follows from this and (2.9) that $g_{i k}=\langle 0\rangle$ for $k \neq i$ and this, together with (2.8), implies that

$$
(v w)_{i}=v_{i} g_{i i}(w) \quad \forall v, w \in \mathbb{R}^{n}, 1 \leq i \leq n .
$$

For $1 \leq i \leq n$, define a continuous selfmap $f_{i}$ by $f_{i}(x)=g_{i i}(0,0, \ldots, 0, x)$. Since $v w=$ $v\left(0,0, \ldots, 0, w_{n}\right)$, it follows from (2.10) that

$$
(v w)_{i}=v\left(0,0, \ldots, 0, w_{n}\right)=v_{i} g_{i i}\left(0,0, \ldots, 0, w_{n}\right)=v_{i} f_{i}\left(w_{n}\right)
$$

for $1 \leq i \leq n$ and we see that the multiplication on $\mathcal{N}_{n}$ is, indeed, given by (2.6). It follows from (2.11) that

$$
\begin{aligned}
& (u(v w))_{i}=u_{i} f_{i}\left((v w)_{n}\right)=u_{i} f_{i}\left(v_{n} f_{n}\left(w_{n}\right)\right), \\
& ((u v) w)_{i}=(u v)_{i} f_{i}\left(w_{n}\right)=u_{i} f_{i}\left(v_{n}\right) f_{i}\left(w_{n}\right),
\end{aligned}
$$

for all $u, v, w \in \mathbb{R}^{n}$. Let $u_{i}=1, v_{n}=x$, and $w_{n}=y$ in (2.12) and, since $u(v w)=$ $(u v) w$, conclude that

$$
f_{i}\left(x f_{n}(y)\right)=f_{i}(x) f_{i}(y) \quad \forall x, y \in \mathbb{R}, 1 \leq i \leq n,
$$

and we see that (2.5) is satisfied. This concludes the proof. 
Our next task is to characterize those functions which satisfy (2.5). Take $i=n$ in (2.5) and get $f_{n}\left(x, f_{n}(y)\right)=f_{n}(x) f_{n}(y)$ for all $x, y \in \mathbb{R}$ which implies that

$$
f_{n}(r x)=r f_{n}(x) \quad \forall x \in \mathbb{R}, \forall r \in \operatorname{Ran}\left(f_{n}\right),
$$

where $\operatorname{Ran}\left(f_{n}\right)$ denotes the range of $f_{n}$. Such a function was referred to in [3] as a semilinear map and it was shown in [3, Theorem 3.3] that either $f_{n}=\langle 0\rangle, f_{n}=\langle 1\rangle$, or $\operatorname{Ran}\left(f_{n}\right)=\mathbb{R}$, or $\operatorname{Ran}\left(f_{n}\right)=\mathbb{R}^{+}$where $\mathbb{R}^{+}$denotes the nonnegative reals.

LEMMA 2.4. Suppose $f_{n}=\langle 0\rangle$. Then (2.5) is satisfied if and only if either $f_{i}=\langle 0\rangle$ or $f_{i}=\langle 1\rangle$ for $1 \leq i<n$.

Proof. Suppose (2.5) is satisfied. Then $f_{i}(x) f_{i}(y)=f_{i}(0)$ for all $x, y \in \mathbb{R}$. Thus $\left(f_{i}(0)\right)^{2}=f_{i}(0)$ which means that either $f_{i}(0)=0$ or $f_{i}(0)=1$. Since $\left(f_{i}(x)\right)^{2}=f_{i}(0)$ for all $x \in \mathbb{R}$, it readily follows that either $f_{i}=\langle 0\rangle$ or $f_{i}=\langle 1\rangle$ for $1 \leq i \leq n$.

One easily verifies that if either $f_{i}=\langle 0\rangle$ or $f_{i}=\langle 1\rangle$ for $1 \leq i<n$, then (2.5) is satisfied and, with this observation, the proof is complete.

The proof of Lemma 2.5 is similar to the proof of Lemma 2.4 and, for that reason, is omitted.

LEMmA 2.5. Suppose $f_{n}=\langle 1\rangle$. Then (2.5) is satisfied if and only if either $f_{i}=\langle 0\rangle$ or $f_{i}=\langle 1\rangle$ for $1 \leq i<n$.

LEMMA 2.6. Let $h$ be a continuous selfmap of $\mathbb{R}$ and suppose that

$$
h(x+y+b)=h(x)+h(y) \quad \forall x, y \in \mathbb{R},
$$

where $b$ is any fixed real number. Then there exists a real number $r$ such that $h(x)=$ $r x+r b$ for all $x \in \mathbb{R}$.

Proof. We have $h(x+z+b)=h(x)+h(z)$ for all $x, z \in \mathbb{R}$. Let $s=-b$ and $z=y+s$ and the latter transforms into

$$
h(x+y)=h(x)+h(y+s) \quad \forall x, y \in \mathbb{R} .
$$

Let $h(2 s)=t$ and it then follows from (2.16) that $h(x+s)=h(x)+t$ or, equivalently, $h(y+s)=h(y)+t$ for all $y \in \mathbb{R}$. We can now rewrite (2.16) as

$$
h(x+y)=h(x)+h(y)+t \quad \forall x, y \in \mathbb{R} .
$$

One uses induction on (2.17) to show that

$$
h(m x)=m h(x)+(m-1) t .
$$

For any nonzero integer $n$, let $x=1 / n$ in (2.18) and get

$$
h\left(\frac{m}{n}\right)=m h\left(\frac{1}{n}\right)+(m-1) t .
$$

Let $m=1$ and $h(1)=a$ in (2.19) and get

$$
h\left(\frac{1}{n}\right)=\frac{a+(1-n) t}{n} .
$$


From (2.19) and (2.20), we get

$$
h\left(\frac{m}{n}\right)=\frac{m}{n}(a+(1-n) t)=\frac{m}{n}(a+t)-t .
$$

Let $r=a+t$ and conclude from (2.21) that $h(x)=r x-t$ for all rational numbers. Since $h$ is continuous, we conclude that

$$
h(x)=r x-t \quad \forall x \in \mathbb{R} .
$$

We use the latter fact and (2.15) to get

$$
r x+r y+r b-t=h(x+y+b)=h(x)+h(y)=r x-t+r y-t
$$

for all $x, y \in \mathbb{R}$ which implies that $r b=-t$. It follows from this and (2.22) that $h(x)=$ $r x+r b$ for all $x \in \mathbb{R}$ and the proof is complete.

Recall that for any positive number $r$, we define $0^{r}=0$.

LEMMA 2.7. Let a be a nonzero real number and let $h$ be a nonconstant continuous selfmap of $\mathbb{R}$. If $a<0$ and

$$
h(\operatorname{axy})=h(x) h(y) \quad \forall x \in \mathbb{R}, \forall y \leq 0,
$$

then there exists a positive real number $r$ such that

$$
h(x)=(a x)^{r} \quad \forall x \leq 0 .
$$

If $a>0$ and

$$
h(\operatorname{axy})=h(x) h(y) \quad \forall x \in \mathbb{R}, \forall y \geq 0,
$$

then there exists a positive real number $r$ such that

$$
h(x)=(a x)^{r}, \quad \forall x \geq 0 .
$$

Proof. Suppose first that $a<0$ and $h$ satisfies (2.24). Suppose further that $h(y)=$ 0 for some $y<0$. Then (2.24) implies that $h(a x y)=0$ for all $x \in \mathbb{R}$ which results in the contradiction that $h=\langle 0\rangle$. Consequently, $h(y) \neq 0$ whenever $y<0$. It also follows from (2.24) that $h\left(a x^{2}\right)=(h(x))^{2}$ for all $x<0$. Since every negative number is of the form $a x^{2}$ for appropriate $x$, we conclude that $h(-\infty, 0) \subseteq(0, \infty)$. Define a homeomorphism $\varphi$ from $\mathbb{R}$ onto $(-\infty, 0)$ by $\varphi(x)=-e^{x}$ and define a homeomorphism $\psi$ from $(0, \infty)$ onto $\mathbb{R}$ by $\psi(x)=\ln x$ and let $\alpha=\psi \circ h \circ \varphi$. In view of our previous observations, $\alpha$ is a continuous selfmap of $\mathbb{R}$. Let $b=\ln (-a)$. Then

$$
\begin{aligned}
\alpha(x+y+b) & =\psi\left(h\left(-e^{x+y+b}\right)\right)=\psi\left(h\left(-e^{b}\left(-e^{x}\right)\left(-e^{y}\right)\right)\right) \\
& =\psi\left(h\left(a\left(-e^{x}\right)\left(-e^{y}\right)\right)\right)=\psi\left(h\left(-e^{x}\right) h\left(-e^{y}\right)\right) \\
& =\psi\left(h\left(-e^{x}\right)\right)+\psi\left(h\left(-e^{y}\right)\right)=\alpha(x)+\alpha(y)
\end{aligned}
$$

for all $x, y \in \mathbb{R}$ and it follows from Lemma 2.5 that $\alpha(x)=r x+r b$ for some $r \in \mathbb{R}$. Now, $h=\psi^{-1} \circ \alpha \varphi^{-1}$ and $\psi^{-1}(x)=e^{x}$ while $\varphi^{-1}(x)=\ln (-x)$. Let $s=e^{r b}$ and for $x<0$, we have

$$
\begin{aligned}
h(x) & =\psi^{-1} \circ \alpha(\ln (-x))=\psi^{-1}(r \ln (-x)+r b) \\
& =\psi^{-1}(r \ln |x|+r b)=e^{r b}\left(e^{\ln |x|}\right)^{r}=s|x|^{r} .
\end{aligned}
$$


Evidently $s>0$. Suppose $r<0$. Then

$$
\lim _{x \rightarrow 0^{-}} h(x)=\lim _{x \rightarrow 0^{-}} s|x|^{r}=\infty,
$$

which contradicts the continuity of $h$ at zero. Thus, $r \geq 0$. Suppose $r=0$. Then $h(x)=s$ for all $x \leq 0$. Let $x=y=-1$ in (2.24) and get $s=h(a(-1)(-1))=(h(-1))^{2}=$ $s^{2}$. This means that $s=1$ since $s>0$ and we now have $h(x)=1$ for all $x \leq 0$. Next, let $x=1$ and let $y<0$ in (2.24). Then $h(a y)=h(a \cdot 1 \cdot y)=h(1) h(y)=h(1)$. Since every positive number is of the form $a y$ for some negative number $y$, we conclude that $h(x)=h(1)$ for all $x>0$. Thus, we have $h(x)=1$ for all $x \in \mathbb{R}$ but this contradicts the fact that $h$ is a nonconstant map. Hence, we must conclude that $r \neq 0$ which means $r>0$. Take $x=y=-1$ in (2.24) and use (2.29) to get

$$
s|a|^{r}=h(a)=h(a(-1)(-1))=(h(-1))^{2}=s^{2},
$$

which implies that $s=|a|^{r}$. It follows from this fact and (2.29) that (2.25) holds.

The case where $a>0$ is quite similar to the previous case so we will be content with making a few remarks and omitting most of the details. Suppose $h(x)=0$ for some $x>0$. Then, for all $y>0$, we have $h(a x y)=h(x) h(y)=0$ and we conclude that $h(x)=0$ for all $x \geq 0$. Next, let $x<0$ and we have $0=h\left(a x^{2}\right)=h(x) h(x)$. This means that $h(x)=0$ for all $x$ which contradicts the fact that $h$ is nonconstant. Therefore, $h(x) \neq 0$ for $x>0$. Moreover, for any $x>0,(2.26)$ assures that $h\left(a x^{2}\right)=(h(x))^{2}$ and it follows that $h(0, \infty) \subseteq h(0, \infty)$. This time, define a homeomorphism $\varphi$ from $\mathbb{R}$ onto $(0, \infty)$ by $\varphi(x)=e^{x}$ and a homeomorphism $\psi$ from $(0, \infty)$ onto $\mathbb{R}$ by $\psi(x)=\ln x$. Again let $\alpha=\psi \circ h \circ \varphi$ and one easily verifies that $\alpha(x+y+b)=\alpha(x)+\alpha(y)$ for all $x, y \in \mathbb{R}$ where $b=\ln a$. As before, it follows from Lemma 2.6 that $\psi \circ h \circ \varphi(x)=$ $\alpha(x)=r x+r b$ for some $r \in \mathbb{R}$. With a few minor modifications of the arguments used in the previous case, one shows that the latter implies that (2.27) holds.

LEMMA 2.8. Let $a \neq 0$ and let $h$ be a nonconstant continuous selfmap of $\mathbb{R}$. Then $h$ satisfies the functional equation

$$
h(a x y)=h(x) h(y) \quad \forall x, y \in \mathbb{R}
$$

if and only if there exists a positive number $r$ such that either

$$
h(x)=|a x|^{r} \quad \forall x \in \mathbb{R},
$$

or

$$
h(x)= \begin{cases}(a x)^{r} & \text { for } a x \geq 0, \\ -|a x|^{r} & \text { for } a x<0 .\end{cases}
$$

Proof. Consider first the case where $a<0$. It follows from Lemma 2.7 that

$$
h(x)=(a x)^{r} \quad \forall x \leq 0 .
$$

Next, let $x>0$ and $y=1$ in (2.32). Then $a x<0$ and we appeal to (2.32) and (2.35) to get

$$
\left(a^{2} x\right)^{r}=h(a x)=h(a x 1)=h(x) h(1) .
$$


Now $h(1) \neq 0$ since $\left(a^{2} x\right)^{r} \neq 0$ whenever $x \neq 0$ so we let $p=\left(a^{2}\right)^{r} / h(1)$ and from (2.36), we conclude that $h(x)=p x^{r}$ for $x>0$. Take $x=y=1$ in (2.32) and since $a<0$, we get

$$
|a|^{2 r}=\left(a^{2}\right)^{r}=h(a)=h(a \cdot 1 \cdot 1)=(h(1))^{2} .
$$

It follows from (2.37) that either $h(1)=|a|^{r}$ or $h(1)=-|a|^{r}$.

CASE $1\left(h(1)=|a|^{r}\right)$. Then $p=\left(a^{2}\right) r / h(1)=\left(|a|^{r}\right)^{2} /|a|^{r}=|a|^{r}$ and we conclude that $h(x)=p x^{r}=|a|^{r} x^{r}=|a x|^{r}$ for all $x>0$. It follows from this and (2.35) that $h(x)=|a x|^{r}$ for all $x \in \mathbb{R}$. That is, the function $h$ is given by (2.33).

CASE $2\left(h(1)=-|a|^{r}\right)$. Then $p=\left(a^{2}\right)^{r} / h(1)=\left(|a|^{r}\right)^{2} /-|a|^{r}=-|a|^{r}$ and we see that $h(x)=p x^{r}=-|a|^{r} x^{r}=-|a x|^{r}$ for $x>0$. Since $a<0$, it follows from this fact and (2.35) that, in this case, the function $h$ is given by (2.34) and this verifies the lemma for the case where $a<0$. The case where $a>0$ is quite similar to the previous case and for that reason we omit the details.

LEMMA 2.9. Let $a$ be a nonpositive real number and $b$ a nonnegative real number such that $a^{2}+b^{2} \neq 0$ and let $h$ be a nonconstant continuous selfmap of $\mathbb{R}$. Then

$$
\begin{array}{ll}
h(\text { axy })=h(x) h(y) & \forall x \in \mathbb{R}, y \leq 0, \\
h(b x y)=h(x) h(y) & \forall x \in \mathbb{R}, y \geq 0,
\end{array}
$$

if and only if there exists an $r>0$ such that

$$
h(x)= \begin{cases}(a x)^{r} & \text { for } x \leq 0, \\ (b x)^{r} & \text { for } x \geq 0 .\end{cases}
$$

Proof. Suppose that (2.38) and (2.39) are both satisfied. We first consider the case where $a \neq 0 \neq b$. It then follows immediately from Lemma 2.7 that there exist positive real numbers $r$ and $s$ such that

$$
h(x)= \begin{cases}(a x)^{r} & \text { for } x \leq 0, \\ (b x)^{s} & \text { for } x \geq 0 .\end{cases}
$$

Take $x=1$ and $y=-1$ and it follows from (2.38) and (2.41) that

$$
(-a)^{s} b^{s}=(-a b)^{s}=f(-a)=f(a \cdot 1 \cdot(-1))=f(1) f(-1)=b^{s}(-a)^{r}
$$

which readily implies that $(-a)^{s}=(-a)^{r}$ which, in turn, implies that $r=s$. Thus, $h$ is given by (2.40). It is a routine matter to check that if $h$ is given by (2.40), then it satisfies both (2.38) and (2.39) and we omit the details.

We next consider the case where $h$ satisfies (2.38) and (2.39) and $a=0$. Then $b \neq 0$ and it follows from (2.39) and Lemma 2.7 that there exists a positive number $r$ such that $h(x)=(b x)^{r}$ for all $x \geq 0$. It follows from (2.38) that $h(0)=h(x) h(y)$ for all $x \in \mathbb{R}$ and all $y \leq 0$. Since $h(0)=(h(0))^{2}$, we have either $h(0)=0$ or $h(0)=1$. Suppose $h(0)=1$. Then $h(x) h(y)=1$ for all $x \in \mathbb{R}$ and $y \leq 0$. Take $y=0$ and conclude that $h(x)=1$ for all $x \in \mathbb{R}$. But this contradicts the fact that $h$ is nonconstant. 
Consequently, we must have $h(0)=0$ which means $h(x) h(y)=0$ for all $x \in \mathbb{R}$ and all $y \leq 0$. Let $x=1$ and get $b^{r} h(y)=0$ for all $y \leq 0$. Thus, $h(y)=0$ for all $y \leq 0$ and we have shown that $f$ is given by (2.40) in this case also. It is immediate that if $h$ satisfies (2.40) and $a=0$, then $h$ satisfies both (2.38) and (2.39). The case where $b=0$ is very similar to the preceding case and we say no more about it.

THEOREM 2.10. The functions $f_{i}(1 \leq i \leq n)$ satisfy (2.5) if and only if

$$
f_{i}=\langle 0\rangle \text { or } f_{i}=\langle 1\rangle \text { for } 1 \leq i \leq n,
$$

or there exist a nonzero number $a$ and positive numbers $r_{i}(1 \leq i<n)$ such that $f_{n}(x)=a x$ and either $f_{i}(x)=|a x|^{r_{i}}$ or

$$
f_{i}(x)= \begin{cases}(a x)^{r_{i}} & \text { for } a x \geq 0, \\ -|a x|^{r_{i}} & \text { for } a x<0,\end{cases}
$$

or there exist an $a \leq 0, a b \geq 0$ with $a^{2}+b^{2} \neq 0$ and $r_{i}>0(1 \leq i<n)$ such that

$$
\begin{aligned}
& f_{n}(x)= \begin{cases}a x & \text { for } x \leq 0, \\
b x & \text { for } x>0,\end{cases} \\
& f_{i}(x)= \begin{cases}(a x)^{r_{i}} & \text { for } x \leq 0, \\
(b x)^{r_{i}} & \text { for } x>0,\end{cases}
\end{aligned}
$$

for $1 \leq i<n$.

Proof. It is immediate that the functions $\left\{f_{i}\right\}_{i=1}^{n}$ satisfy (2.5) if (2.43) holds and it is a routine matter to show that if $f_{n}(x)=a x$ for some $a \neq 0$ and there exist $n-1$ positive numbers $\left\{r_{i}\right\}_{i=1}^{n-1}$ such that either $f_{i}(x)=|a x|^{r_{i}}$ for $1 \leq i<n$ or $f_{i}(x)$ is given by (2.44), then the functions $\left\{f_{i}\right\}_{i=1}^{n}$ satisfy (2.5). Similarly, it follows in a routine manner that if $f_{n}$ and $f_{i}(1 \leq i<n)$ are given by (2.45) and (2.46), respectively, then the functions $\left\{f_{i}\right\}_{i=1}^{n}$ again satisfy (2.5).

Suppose, conversely, that the functions $\left\{f_{i}\right\}_{i=1}^{n}$ satisfy (2.5). We must show that they must be one of the families described in the statement of the theorem. First of all, we observed following the proof of Lemma 2.3 that by taking $i=n$ in (2.5), we get $f_{n}(r x)=r f_{n}(x)$ for all $x \in \mathbb{R}$ and all $r \in \operatorname{Ran}\left(f_{n}\right)$ and it was shown in [3, Theorem 3.3] that either $f_{n}=\langle 0\rangle, f_{n}=\langle 1\rangle$, or $\operatorname{Ran}\left(f_{n}\right)=\mathbb{R}$ or $\operatorname{Ran}\left(f_{n}\right)=\mathbb{R}^{+}$.

CASE $3\left(f_{n}=\langle 0\rangle\right.$ or $\left.f_{n}=\langle 1\rangle\right)$. It follows from Lemmas 2.4 and 2.5 that $f_{i}=\langle 0\rangle$ or $f_{i}=\langle 1\rangle$ for $1 \leq i \leq n$ and thus, (2.43) is satisfied.

CASE $4\left(\operatorname{Ran}\left(f_{n}\right)=\mathbb{R}\right)$. In this case, we have $f_{n}(x y)=x f_{n}(y)$ for all $x, y \in \mathbb{R}$. Let $y=1$, let $f(1)=a$ and conclude that $f_{n}(x)=a x$ for all $x \in \mathbb{R}$. Evidently, $a \neq 0$ since $\operatorname{Ran}\left(f_{n}\right)=\mathbb{R}$. Since (2.5) is satisfied, we have $f_{i}(a x y)=f_{i}(x) f_{i}(y)$ for all $x, y \in \mathbb{R}$ and it follows from Lemma 2.8 that there exists a positive number $r_{i}$ such that either $f_{i}(x)=|a x|^{r_{i}}$ for all $x \in \mathbb{R}$ or

$$
f_{i}(x)= \begin{cases}(a x)^{r_{i}} & \text { for } a x \geq 0 \\ -|a x|^{r_{i}} & \text { for } a x<0\end{cases}
$$

for all $x \in \mathbb{R}$. 
CASE $5\left(\operatorname{Ran}\left(f_{n}\right)=\mathbb{R}^{+}\right)$. In this case, we have $f_{n}(x y)=x f_{n}(y)$ for all $x \geq 0$ and all $y \in \mathbb{R}$. Take $y=1$ and $b=f_{n}(1)$ and conclude that $f_{n}(x)=b x$ for $x \geq 0$. Next, let $x<0$, take $y=-1$, let $a=-f_{n}(-1)$ and conclude that $f_{n}(x)=f_{n}((-x)(-1))=$ $-x f_{n}(-1)=a x$. Evidently, $a \leq 0, b \geq 0$, and $a^{2}+b^{2} \neq 0$ since $\operatorname{Ran}\left(f_{n}\right)=\mathbb{R}^{+}$. In this case, $f_{n}$ satisfies (2.45) and it follows from (2.5) that $f_{i}(a x y)=f_{i}(x) f_{i}(y)$ for $y \leq 0$ and $f_{i}(b x y)=f_{i}(x) f_{i}(y)$ for $y \leq 0$. This, in view of Lemma 2.9, implies that each $f_{i}$, $1 \leq i<n$, is given by (2.46). This completes the proof.

Our next result is an immediate consequence of Lemma 2.3 and Theorem 2.10. Recall that $K_{i}=\left\{v \in \mathbb{R}^{n}: v_{j}=0\right.$ for $\left.j \neq i\right\}$.

THEOREM 2.11. Let $\mathcal{N}_{n}$ be an $n$-dimensional Euclidean nearring. Then each $K_{i}, 1 \leq$ $i \leq n$, is a right ideal of $\mathcal{N}_{n}$ and $v w=v\left(0,0, \ldots, 0, w_{n}\right)$ if and only if the multiplication in $\mathcal{N}_{n}$ is given by one of the following:

$$
\begin{aligned}
& (v w)_{i}=0 \quad \text { or } \quad(v w)_{i}=v_{i}, \quad \text { for } 1 \leq i \leq n, \\
& (v w)_{i}=v_{i}\left|a w_{n}\right|^{r_{i}} \quad \text { for } i \neq n, \quad(v w)_{n}=a v_{n} w_{n},
\end{aligned}
$$

where $a \neq 0$ and $r_{i}>0$ for each $i$,

$$
(v w)_{i}= \begin{cases}v_{i}\left(a w_{n}\right)^{r_{i}} & \text { for } a w_{n} \geq 0, \\ -v_{i}\left|a w_{n}\right|^{r_{i}} & \text { for } a w_{n}<0,\end{cases}
$$

for $i \neq n$ and $(v w)_{n}=a v_{n} w_{n}$ where $a \neq 0$ and $r_{i}>0$,

$$
\begin{aligned}
& (v w)_{i}= \begin{cases}v_{i}\left(a w_{n}\right)^{r_{i}} & \text { for } w_{n} \leq 0, i \neq n, \\
v_{i}\left(b w_{n}\right)^{r_{i}} & \text { for } w_{n}>0, i \neq n,\end{cases} \\
& (v w)_{n}= \begin{cases}a v_{n} w_{n} & \text { for } w_{n} \leq 0, \\
b v_{n} w_{n} & \text { for } w_{n}>0,\end{cases}
\end{aligned}
$$

where $r_{i}>0, a \leq 0, b \geq 0$, and $a^{2}+b^{2} \neq 0$.

Proof OF TheOrem 2.2. Suppose first that $\mathcal{N}_{n}$ is a Euclidean nearring which is isomorphic to a nearring $\mathcal{N}_{n 1}$ whose multiplication is given by any one of (2.1), (2.2), (2.3), and (2.4) inclusive. It is immediate that each $K_{i}, 1 \leq i \leq n$ is a right ideal of $\mathcal{N}_{n 1}$. For each $w \in \mathcal{N}_{n 1}$, let $w^{i}$ be the vector such that $w_{i}^{i}=w_{i}$ and $w_{j}^{i}=0$ for $j \neq i$. Then $w^{i} \in K_{i}, w=w^{1}+w^{2}+\cdots+w^{n}$, and $v w=v w^{n}$ for all $v \in \mathcal{N}_{n 1}$. That is, $\mathcal{N}_{n 1}$ is a linear right ideal nearring and so is $\mathcal{N}_{n}$ since any isomorphism from $\mathcal{N}_{n 1}$ onto $\mathcal{N}_{n}$ is a linear automorphism of $\mathbb{R}^{n}$.

Suppose, conversely, that $\mathcal{N}_{n}$ is a linear right ideal nearring with linear right ideals $J_{i}, 1 \leq i \leq n$. Let $\varphi$ be any linear automorphism of $\mathbb{R}^{n}$ such that $\varphi\left[K_{i}\right]=J_{i}$ and define a multiplication $*$ on $\left(\mathbb{R}^{n},+\right)$ by $v * w=\varphi^{-1}(\varphi(v) \varphi(w))$ where $\varphi(v) \varphi(w)$ is the product of $\varphi(v)$ and $\varphi(w)$ in the nearring $\mathcal{N}_{n}$. With this multiplication, $\left(\mathbb{R}^{n},+, *\right)$ is a nearring which we denote by $\mathcal{N}_{n 1}$ hereafter. Moreover, $\varphi$ is an isomorphism from $\mathcal{N}_{n 1}$ onto $\mathcal{N}_{n}$. Consequently, each $K_{i}$ is a right ideal of $\mathcal{N}_{n 1}$. According to Definition 2.1, for each $w \in \mathcal{N}_{n 1}$, there exist $w^{i} \in J_{i}, 1 \leq i \leq n$, such that $\varphi(w)=w^{1}+w^{2}+\cdots+w^{n}$ and 
$\varphi(v) \varphi(w)=\varphi\left(w^{n}\right)$. Since $w=\varphi^{-1}\left(w^{1}\right)+\varphi^{-1}\left(w^{2}\right)+\cdots+\varphi^{-1}\left(w^{n}\right)$ and $\varphi^{-1}\left(w^{i}\right) \in$ $K_{i}$ for $1 \leq i \leq n$, it readily follows that $\left(\varphi^{-1}\left(w^{i}\right)\right)_{i}=w_{i}$ for each $i$ and $\left(\varphi^{-1}\left(w^{i}\right)\right)_{j}=0$ for $j \neq i$. Consequently, all this implies that

$$
v * w=\varphi^{-1}(\varphi(v) \varphi(w))=v * \varphi^{-1}\left(w^{n}\right)=v *\left(0,0, \ldots, 0, w_{n}\right)
$$

and it follows from Theorem 2.11 that the multiplication in $\mathcal{N}_{n 1}$ is given by one of (2.48) to (2.51). The proof will be complete once we show that the nearring whose multiplication is given by (2.49) is isomorphic to the one whose multiplication is given by (2.2) and the nearring whose multiplication is given by (2.50) is isomorphic to the nearring whose multiplication is given by (2.3). Denote by $\mathcal{N}_{n 1}$ the nearring whose multiplication is given by (2.2) and denote by $\mathcal{N}_{n 2}$ the nearring whose multiplication is given by (2.49). The multiplication in both cases will be denoted by juxtaposition. Since $a \neq 0$, we are able to define a bijection $\varphi$ from $\mathcal{N}_{n 1}$ onto $\mathcal{N}_{n 2}$ by

$$
(\varphi(v))_{i}=\frac{v_{i}}{|a|^{r_{i}}} \quad \text { for } 1 \leq i<n, \quad(\varphi(v))_{n}=\frac{v_{n}}{a} .
$$

It is immediate that $\varphi$ is an additive isomorphism. As for multiplication, for $1 \leq i<n$ we have

$$
\begin{gathered}
(\varphi(v w))_{i}=\frac{(v w)_{i}}{|a|^{r_{i}}}=\frac{v_{i}\left|w_{n}\right|^{r_{i}}}{|a|^{r_{i}}} \\
(\varphi(v) \varphi(w))_{i}=(\varphi(v))_{i}\left|a(\varphi(w))_{n}\right|^{r_{i}}=\frac{v_{i}}{|a|^{r_{i}}}\left|a \frac{w_{n}}{a}\right|^{r_{i}}=\frac{v_{i}\left|w_{n}\right|^{r_{i}}}{|a|^{r_{i}}} .
\end{gathered}
$$

In addition to this, we have

$$
(\varphi(v w))_{n}=\frac{v_{n} w_{n}}{a}=a(\varphi(v))_{n}(\varphi(w))_{n}=(\varphi(v) \varphi(w))_{n}
$$

and it follows from (2.54), (2.55), and (2.56) that $\varphi$ is an isomorphism from $\mathcal{N}_{n 1}$ onto $\mathcal{N}_{n 2}$.

This time, let $\mathcal{N}_{n 1}$ be a nearring whose multiplication is given by (2.3) and let $\mathcal{N}_{n 2}$ be a nearring whose multiplication is given by (2.50). Again, define a bijection from $\mathcal{N}_{n 1}$ onto $\mathcal{N}_{n 2}$ just as in (2.53). Here, there are a number of cases to consider for a product $v w$ depending upon whether $a<0, a>0, w_{n}<0$, or $w_{n} \geq 0$. Consider the case where $a<0$ and $w_{n}<0$. It then follows that for $1 \leq i<n$, we have

$$
(\varphi(v w))_{i}=\frac{(v w)_{i}}{|a|^{r_{i}}}=-\frac{v_{i}\left|w_{n}\right|^{r_{i}}}{|a|^{r_{i}}} .
$$

Since $a(\varphi(w))_{n}=w_{n}<0$, we also have

$$
(\varphi(v) \varphi(w))_{i}=-(\varphi(v))_{i}\left|a(\varphi(w))_{n}\right|^{r_{i}}=-\frac{v_{i}\left|w_{n}\right|^{r_{i}}}{|a|^{r_{i}}} .
$$

In addition, we have

$$
(\varphi(v w))_{n}=\frac{(v w)_{n}}{a}=\frac{v_{n} w_{n}}{a}=a(\varphi(v))_{n}(\varphi(w))_{n}=(\varphi(v) \varphi(w))_{n},
$$

and it follows from (2.57), (2.58), and (2.59) that $\varphi(v w)=\varphi(v) \varphi(w)$ whenever $a<0$ and $w_{n}<0$. The remaining cases follow in much the same manner and, with this observation, we conclude the proof. 
COROLlARY 2.12. A two-dimensional Euclidean nearring $\mathcal{N}_{2}$ has two distinct nonzero proper closed, connected right ideals $J_{1}$ and $J_{2}$ such that for every $w \in \mathcal{N}_{2}$ there exist $w^{1} \in J_{1}$ and $w^{2} \in J_{2}$ such that $w=w^{1}+w^{2}$ and $v w=v w^{2}$ for all $v \in \mathcal{N}_{2}$ if and only if $\mathcal{N}_{2}$ is isomorphic to one of the seven two-dimensional Euclidean nearrings whose multiplications follow:

$$
\begin{aligned}
& v w=v \quad \forall v, w \in \mathcal{N}_{2}, \\
& v w=(0,0) \quad \forall v, w \in \mathcal{N}_{2}, \\
& v w=\left(v_{1}, 0\right) \quad \forall v, w \in \mathcal{N}_{2}, \\
& v w=\left(0, v_{2}\right) \quad \forall v, w \in \mathcal{N}_{2}, \\
& v w=\left(v_{1}\left|w_{2}\right|^{r}, v_{2} w_{2}\right) \quad \forall v, w \in \mathcal{N}_{2} \text {, } \\
& v w= \begin{cases}\left(v_{1} w_{2}^{r}, v_{2} w_{2}\right) & \text { for } w_{2} \geq 0, \\
\left(-v_{1}\left|w_{2}\right|^{r}, v_{2} w_{2}\right) & \text { for } w_{2}<0,\end{cases} \\
& v w= \begin{cases}\left(v_{1}\left(a w_{2}\right)^{r}, a v_{2} w_{2}\right) & \text { for } w_{2} \geq 0, \\
\left(v_{1}\left(b w_{2}\right)^{r}, b v_{2} w_{2}\right) & \text { for } w_{2}>0,\end{cases}
\end{aligned}
$$

where $r>0$ in each case, $a \leq 0, b \geq 0$, and $a^{2}+b^{2} \neq 0$.

Proof. Suppose that the multiplication on a two-dimensional Euclidean nearring $\mathcal{N}$ is given by any one of (2.60) inclusive. Then $K_{1}=\left\{v \in \mathcal{N}_{n}: v_{2}=0\right\}$ and $K_{2}=\left\{v \in \mathcal{N}_{n}\right.$ : $\left.v_{1}=0\right\}$ are two distinct proper closed, connected right ideals of $\mathcal{N}$. For any $w \in \mathcal{N}_{n}$, let $w^{1}=\left(w_{1}, 0\right)$ and $w^{2}=\left(0, w_{2}\right)$. Then $w^{i} \in K_{i}, w=w^{1}+w^{2}$, and $v w=v w^{2}$ for all $v \in \mathcal{N}$. Thus, if $\mathcal{N}_{2}$ is isomorphic to $\mathcal{N}$, then $\mathcal{N}_{2}$ contains two distinct nonzero proper closed, connected right ideals $J_{1}$ and $J_{2}$ such that for every $w \in \mathcal{N}_{2}$ there exist $w^{1} \in J_{1}$ and $w_{2} \in J_{2}$ such that $w=w^{1}+w^{2}$ and $v w=v w_{2}$ for all $v \in \mathcal{N}_{2}$.

Suppose, conversely, that a two-dimensional Euclidean nearring $\mathcal{N}_{2}$ has two distinct nonzero proper closed, connected right ideals $J_{1}$ and $J_{2}$ such that for every $w \in \mathcal{N}_{2}$ there exist $w^{1} \in J_{1}$ and $w_{2} \in J_{2}$ such that $w=w^{1}+w^{2}$ and $v * w=v * w_{2}$ (it is convenient to denote the multiplication in $\mathcal{N}_{2}$ by $*$ ) for all $v \in \mathcal{N}_{2}$. Since the additive subgroup $J_{1}^{+}$of the ideal $J_{1}$ is a closed, connected nonzero subgroup of $\mathbb{R}^{2}$, it follows from [1, Proposition 3, page 71] that $J_{1}^{+}$contains a one-dimensional linear subspace $L_{1}$ of $\mathbb{R}^{2}$. According to one of the statements preceding [1, Proposition 3, page 71], $J_{1}^{+}$ is isomorphic to $\mathbb{R}^{p} \times \mathbb{Z}^{q}$ where $\mathbb{Z}$ is the group of integers and $0 \leq p+q \leq 2$. But $q=0$ since $J_{1}^{+}$is connected and $p=1$ since $J_{1}^{+}$is a proper nonzero subgroup of $\mathbb{R}^{2}$. That is, $J_{1}$ is a linear subspace of $\mathbb{R}^{2}$ and, of course, the same is true of $J_{2}$. Thus, $\mathcal{N}_{2}$ is a linear right ideal nearring and it now follows from Theorem 2.2 that $\mathcal{N}_{2}$ is isomorphic to one of the seven two-dimensional Euclidean nearrings whose multiplications are given by (2.60).

\section{REFERENCES}

[1] N. Bourbaki, Elements of Mathematics. General Topology. Part 2, Hermann and AddisonWesley, Paris and Massachusetts, 1966. MR 34\#5044b. Zbl 301.54002.

[2] J. R. Clay, Nearrings. Geneses and Applications, Oxford Science Publications, Oxford University Press, New York, 1992. MR 94b:16001. Zbl 790.16034. 
[3] K. D. Magill Jr., Topological nearrings whose additive groups are Euclidean, Monatsh. Math. 119 (1995), no. 4, 281-301. MR 96c:16058. Zbl 830.16032.

[4] J. D. P. Meldrum, Near-rings and Their Links with Groups, Research Notes in Mathematics, vol. 134, Pitman, Massachusetts, 1985. MR 88a:16068. Zbl 658.16029.

[5] G. Pilz, Near-rings. The Theory and Its Applications, 2nd ed., North-Holland Math. Studies, vol. 23, North-Holland Publishing, Amsterdam, 1983. MR 85h:16046. Zbl 521.16028.

Kenneth D. Magill JR.: Mathematics Bullding, Rm. 244, SUNY at Buffalo, Buffalo, NY 14260-2900, USA

E-mail address: kdmagi11@acsu. buffa1o.edu 


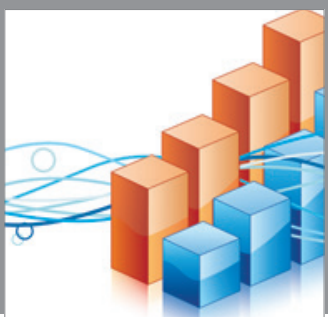

Advances in

Operations Research

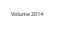

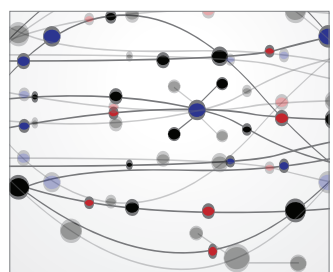

\section{The Scientific} World Journal
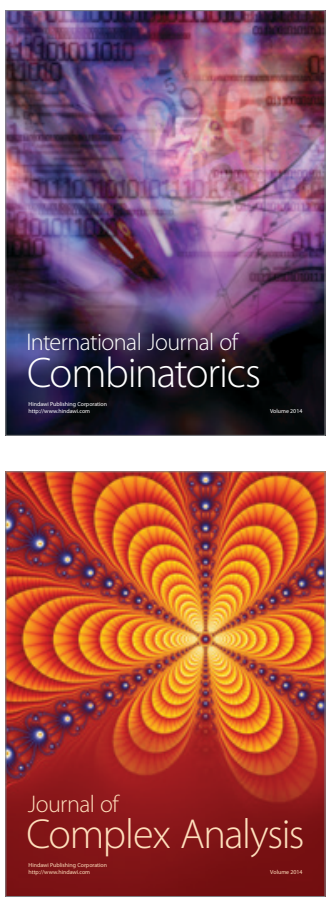

International Journal of

Mathematics and

Mathematical

Sciences
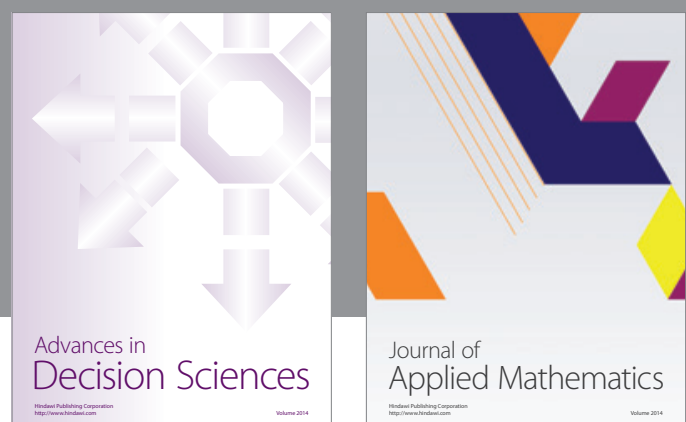

Journal of

Applied Mathematics
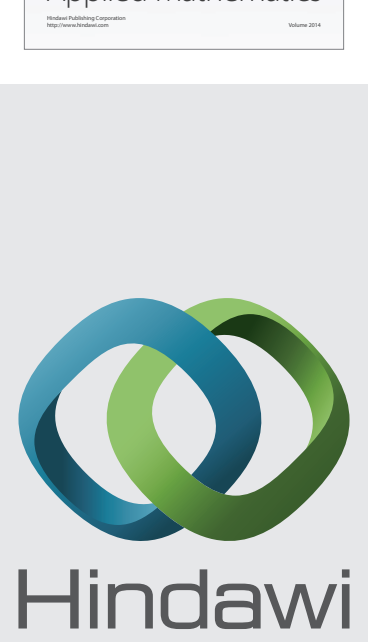

Submit your manuscripts at http://www.hindawi.com
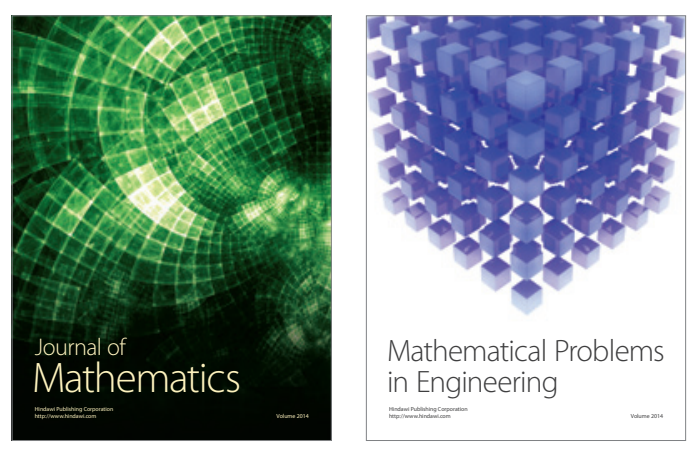

Mathematical Problems in Engineering
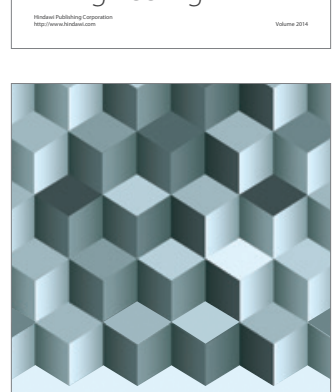

Journal of

Function Spaces
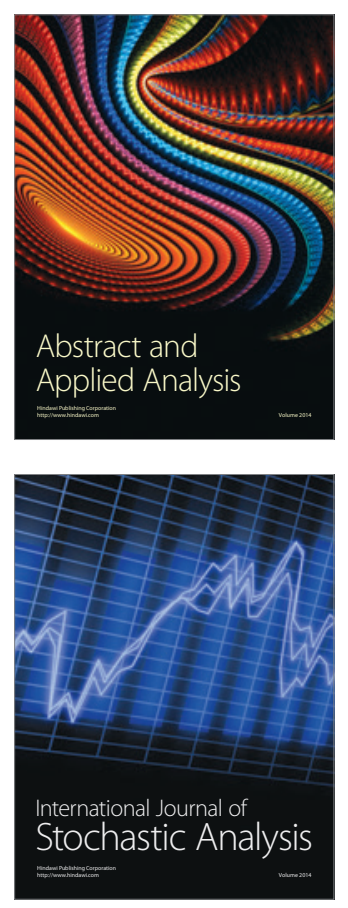

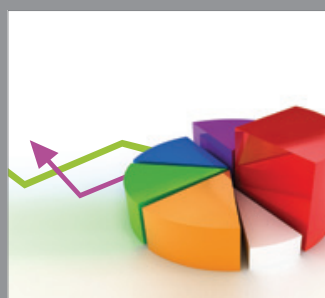

ournal of

Probability and Statistics

Promensencen
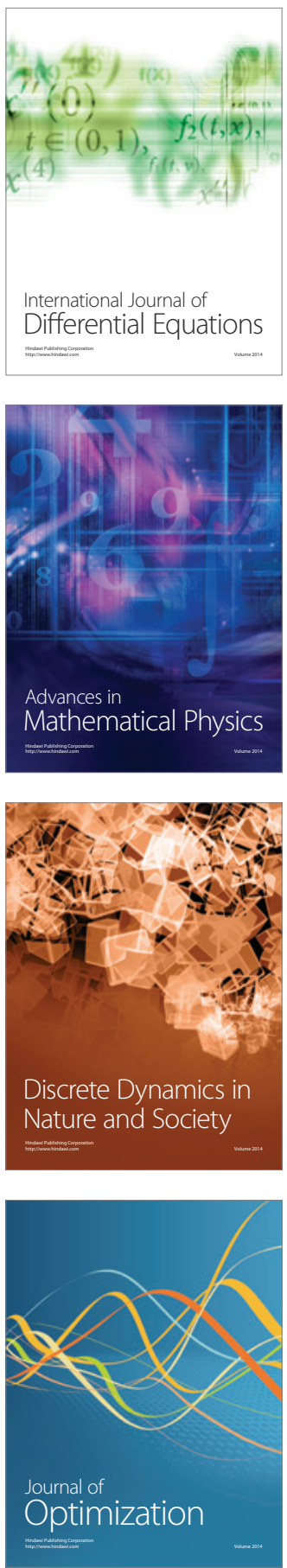